\title{
Design and Evaluation of Techniques for Mobile Interaction with Dynamic NFC-Displays
}

\author{
Gregor Broll, Wolfgang Reithmeier, Paul Holleis, Matthias Wagner \\ DOCOMO Euro-Labs \\ Munich, Germany \\ \{broll, reithmeier, holleis, wagner\}@docomolab-euro.com
}

\begin{abstract}
Near Field Communication (NFC) is a technology for mobile, touch-based interaction with tagged objects that can serve as physical user interfaces (UI). Dynamic NFCdisplays use a grid of NFC-tags as a physical UI and combine it with a projected application UI to enable direct interactions between mobile devices and large screens. In this paper, we explore the design of interaction techniques for dynamic NFC-displays beyond the common touchselect interaction with single tags and evaluate them with an NFC-pinboard prototype. A user study confirms the general preference for the simple touch-select, but also shows that other techniques can provide alternatives, e.g. to implement interactions for which users want to feel more in control.
\end{abstract}

\section{Author Keywords}

Near Field Communication (NFC), physical user interfaces, dynamic NFC-displays, interaction techniques, gestures

\section{ACM Classification Keywords}

H.5.2 [Information Interfaces and Presentation]: User Interfaces - input devices and strategies, interaction styles

\section{General Terms}

Design, Human Factors

\section{INTRODUCTION}

Near Field Communication (NFC) is an emerging technology for mobile interaction with everyday objects [19]. It is a wireless technology for exchanging data over short distances, similar to Radio Frequency Identification (RFID) but targeted at mobile devices. NFC can store digital data on passive tags that can be attached to almost arbitrary objects. Users can retrieve data from a tag by touching it with a reading device, e.g. a mobile phone, or by holding them closely together. The simple, touch-like interaction between NFC-enabled mobile devices, tags, readers or smartcards can facilitate mobile interaction with tagged objects, associated digital information and services in different ways: NFC-tags can be used as physical

Permission to make digital or hard copies of all or part of this work for personal or classroom use is granted without fee provided that copies are not made or distributed for profit or commercial advantage and that copies bear this notice and the full citation on the first page. To copy otherwise, or republish, to post on servers or to redistribute to lists, requires prior specific permission and/or a fee.

TEI'11, January 22-26, 2011, Funchal, Portugal.

Copyright 2011 ACM 978-1-4503-0478-8/11/01..\$10.00. hyperlinks [14] that reduce complex interactions to touching a single tag, e.g. to open a website in a mobile browser. Many applications already use mobile interaction with NFC for mobile payment, ticketing or information retrieval. Other applications map options and features to multiple tags on physical objects from where users can select them directly, instead of browsing nested menus on the small screens of mobile devices. That way, tagged objects can serve as physical user interfaces (UI) that adopt features of mobile UIs and thus complement them. Examples are posters for mobile ticketing [2], tagged maps [10] or control panels for multimedia players [13].

In the next step, dynamic NFC-displays [5] combine the physical interaction with tagged objects and the visual output-capabilities of large screens (Figure 1): They comprise a server that manages the logic of an application and projects its graphical UI onto a grid of NFC-tags that serves as the physical UI. Users can manipulate the content of the projected application UI by touching the tags of the physical UI with an NFC-enabled mobile phone. The phone returns the position of the tag in the grid to the server which updates the application UI according to the interaction.

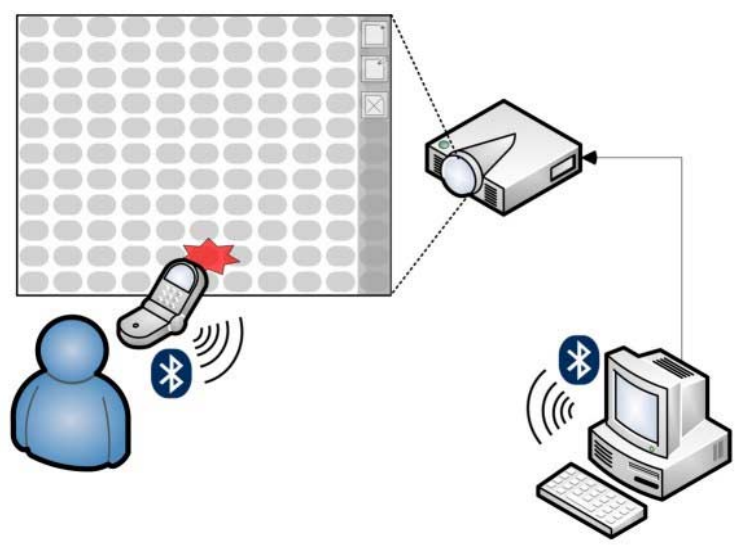

Figure 1. Basic setup of a dynamic NFC-display including the application server, the grid of NFC-tags and the mobile device

In this paper, we investigate interaction techniques for dynamic NFC-displays beyond the common touch-select interaction with single tags. The simplicity of this single-tag interaction is the greatest advantages of mobile interaction with NFC. But as NFC-based physical UIs evolve, their technical capabilities increase and enable new ways to interact with applications and their features. In the 
following sections, we build upon related work and the technology of dynamic NFC-displays to design interaction techniques such as click-select, double-touch, touch\&hold or simple gestures. In order to demonstrate the technical feasibility of these techniques and to evaluate their usability, we have implemented and tested them with an NFC-pinboard prototype. A user study confirms the general preference for the simple touch-select, but also shows that other techniques can partially replace it, e.g. to implement actions for which users want to feel more in control.

\section{RELATED WORK}

Mobile interaction with NFC and RFID has considerably evolved since 1999, when Want et al. [18] tagged everyday objects such as books or business cards with RFID-tags to link them with electronic documents or email-addresses. Since then, NFC and RFID have been used for an increasing number of mobile applications, such as payment (e.g. i-mode FeliCa [7]), ticketing (e.g. Oyster Card [8]), access control, home care or entertainment [16]. In Ubicomp research, NFC and RFID are used to tag physical objects in order to identify them, to link them with digital resources and to facilitate the interaction with them $[2,12]$.

While most applications rely on simple interactions with single NFC-tags, others follow a multi-tag approach to facilitate mobile interactions. They map features to multiple tags on everyday objects, turning them into physical UIs that complement and extend mobile UIs. Examples include posters that comprise multiple tags to let users invoke Web Services for mobile ticketing [2]. Sanchez et al. [13] use a tagged panel to operate a multimedia player whose controls have been mapped to RFID-tags that users can select with their mobile devices. Reilly et al. [10] use RFID-tags on the back of maps to interact with points-of-interest (POI).

In the next step, physical UIs comprise a grid of NFC-tags to provide a completely interactive surface for the manipulation of dynamic application UIs. Opposite to static posters, these tags do not refer to specific items of information, e.g. POIs on a map, but merely indicate their position in the grid. That way, tags can be dynamically mapped to the UI elements of different applications. Vetter et al. [17] and Hardy et al. [5] have created and refined the first dynamic NFC-displays that use a grid of NFC-tags for the interaction with an application UI that is projected onto this interactive surface. Similarly, Ramírez-González et al. [9] have combined a grid of NFC-tags and a projected application UI to build an interactive NFC-panel. Seewoonauth et al. [15] use a grid of tags on the back of a laptop display to enable direct, touch-based interactions.

Dynamic NFC-displays are a prototyping technology that can be used to emulate touch-based interactions between mobile devices and large screens. Their technology allows more direct interactions between mobile devices and items on a screen than other approaches to mobile interaction with public displays that use different input features of mobile devices. WebWall [4] allows users to interact with different features of its virtual displays, e.g. by sending requests through an SMS-gateway. Ballagas et al. [1] present two techniques for mobile interaction with public displays: Sweep uses optical-flow recognition of phonecam images to recognize the movement of a mobile phone and to control a cursor on a display. With Point \& Shoot, users take pictures of a grid of visual markers to identify and select objects on a display. Boring et al. [3] have compared the techniques Scroll (using input from keys or joysticks of mobile phones), Tilt (sensed by an accelerometer) and Move (using optical-flow image recognition) for controlling a cursor on a public display.

Our design of interaction techniques for dynamic NFCdisplays builds upon different existing techniques for mobile interaction with NFC, desktop computing and penbased interaction: Reilly et al. [10] have investigated Clickselect, Path-select, Multi-select, Lasso-select and Menuselect for the interaction with POIs on tagged maps. The dynamic NFC-display of Vetter et al. [17] has supported bounding box selection and path selection. Hardy et al. [5] have added new interaction techniques for their Touch \& Interact-approach: Hovering shows information about a tag on a mobile device when holding them close to each other. The selection of single/multiple tags is achieved by touching them and pressing/holding a key. Polygon-select requires users to hold a key and to touch multiple tags in order to outline an area and to select the tags within this area. Pick-and-drop emulates Rekimoto's Pick-and-drop [11] as it uses the mobile phone to pick up/drop items from/to a screen by touching NFC-tags.

Example interaction techniques from WIMP interfaces in desktop computing include (double-)click, drag\&drop, context menus or the (de)selection of single and multiple items. Li et al. [6] describe techniques for mode switching in pen-based interaction that can be adopted for dynamic NFC-displays. Examples are pressing a button or the Press and Hold technique, which can be translated to touching a dedicated tag or touching a tag for a certain amount of time.

\section{TECHNIQUES AND GESTURES FOR MOBILE INTERACTION WITH DYNAMIC NFC-DISPLAYS}

Dynamic NFC-displays can be used for a wide range of applications at private (e.g. homes), semi-public (e.g. pubs, arcades) and public places (e.g. stations, malls), including information retrieval, interactive advertisements, maps or games. In these and other examples, NFC-enabled mobile phones serve as pointing devices for the interaction with the diverse content of dynamic NFC-displays, including text, pictures, links, maps or custom widgets. Their small, private screens can handle sensitive data, while the large NFC-displays can give a public overview of information. These aspects provide the background for the design of new interaction techniques for dynamic NFC-displays based on related work and the capabilities of their basic technologies. 


\section{Technical Capabilities of Mobile Interaction with NFC} The design, implementation and performance of interaction techniques for dynamic NFC-displays depend on the technical capabilities of reading devices, tags and physical UIs. The selection granularity of dynamic NFC-displays depends on the size of tags, target items and mobile devices. Small tags can increase the resolution of physical UIs and enable more precise interactions. On the other hand, mobile devices occlude tags and items during the interaction with them, making their selection the more difficult the smaller they are.

In addition to this "fat phone"-problem, NFC-enabled mobile phones that are currently available, e.g. the Nokia 6212 , can only read one tag at a time. These devices cannot recognize multiple tags that they occlude during the interaction with one or several items. They also have problems to interact with areas where tags adjoin or overlap. Therefore, the size of tags and items should take the form factor of mobile devices into account.

NFC-enabled devices need a short, but considerable amount of time to read a tag - about 0.5 seconds with currently available devices like the Nokia 6212. This delay prohibits the continuous reading of tags, which is necessary for the recognition of continuous strokes, shapes or gestures. This constraint inevitably divides every multi-tag interaction into a series of discrete interactions with single tags.

\section{Basic Interaction Techniques}

These techniques can be used for simple interactions like the selection of items, triggering actions or activating links.

- Touch-select is the most basic interaction technique. Users touch a tag with a mobile device and remove it after the tag has been recognized. This atomic interaction can be diversified through the combination with input from keyboards, joysticks or sensors.

- Click-select is a touch-select followed by pressing a dedicated key on the mobile device to confirm the selection of a tag. This technique was adopted from [5] and [10] who use it as the default technique to select tags or items that are associated with them.

- Touch\&hold requires users to touch a tag for a period of time that is longer than a regular touch-select, e.g. 2 seconds. This technique was adopted from Press and Hold [6], a technique for pen-based interaction.

- For double-touch-(select), users have to touch the same tag twice in a row, similar to a double-click.

\section{Advanced Interaction Techniques}

Building upon these basic interaction techniques, we have developed some more advanced techniques to manipulate tags and items on dynamic NFC-displays.

Selection of multiple tags and items: The basic interaction techniques can be used to (de)select single or multiple tags or items directly (multi-select). Advanced techniques can adopt basic techniques for the indirect selection of multiple tags or items within an area that is outlined by selecting unoccupied tags.

- Polygon-select was adopted from [5] and can be used to outline areas of arbitrary shapes for the selection of tags and items as long as the first and the last tag that define the outline of an area are the same.

- For bounding-box-select, users have to touch two tags to define a rectangular shape and to select items within it. This technique was adopted from [17].

Drag\&drop: Touch\&drop was adopted from Pick-anddrop $[5,11]$ and uses touch-select or touch\&hold to pick up and drop items with a mobile device. Larger items can also have a dedicated area, e.g. their upper-left corner, for picking them up with a simple touch-select.

Gestures: We have also sounded the capabilities of dynamic NFC-display to implement gestures or shapebased input. Due to the above-mentioned constraints of the basic technology, we have used discrete NFC-touch events to design gestures that are recognized by their shapes and offsets rather than by continuous reading of tags.

- Offset gestures are performed by touching two tags with a specific distance between them, e.g. from the top of an NFC-display to its bottom or from one side to the other (Figure 2a). These gestures have a tolerance level so that users do not have to find the exact offset between two tags. Figure 2a shows alternatives for the same offset gesture within this tolerance level.

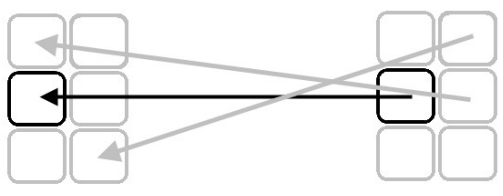

a)

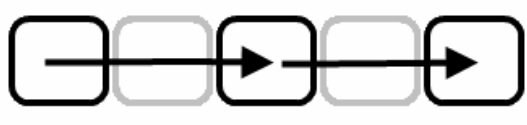

c)

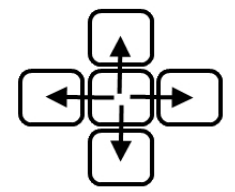

b)

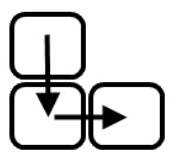

d)
Figure 2. NFC-based gestures: offset gesture (a), one-stroke gestures $(b, c)$ and offset-shape gesture (d)

- To perform one-stroke gestures, users have to touch a fixed number of tags in a fixed direction and with fixed offsets between them, including an offset of zero tags. Examples are gestures that start on one tag, e.g. a specific item, and touch one of the four adjacent tags, e.g. to perform an action on the item (Figure 2b). For another example, users have to touch three tags in a row with an offset of one tag (Figure 2c). 
- Offset-shape gestures extend one-stroke gestures and allow different offsets between their tags. That way, gestures can adopt different shapes (Figure 2d).

\section{THE NFC-PINBOARD}

In order to be able to evaluate the interaction techniques and gestures that we designed for dynamic NFC-displays, we have developed a prototype of an NFC-pinboard. This section presents its features and explains how interaction techniques and gestures can be mapped to them.

\section{Basic Features}

The NFC-pinboard (Figure 3) provides a testbed for the implementation and evaluation of different interaction techniques and gestures for dynamic NFC-displays. It emulates the features of a regular pinboard and can serve as an information hub at semi-public or private places, e.g. companies or homes. Small communities like families can use it to post, view and share digital content from mobile devices like messages, pictures or bookmarks. The NFCpinboard contains different items that can be manipulated by touching the tags of the physical UI. The prototype supports messages and pictures that can be organized in folders. Each item can be opened to expose its contents or closed to save space as an abstract icon. A toolbar contains additional options, e.g. to filter the items on the display.

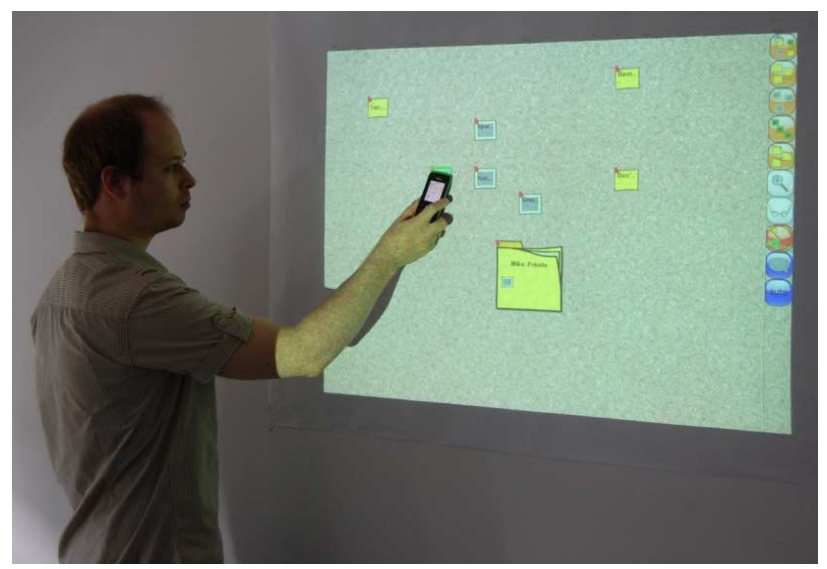

Figure 3. The NFC-pinboard prototype

\section{Application-dependent Interactions}

This section gives an overview of the unique features of the NFC-pinboard and explains which of the previously presented interaction techniques could be used to implement them. Based on this overview, a user study will later investigate the applicability and acceptance of specific interaction techniques for specific application features in the next section.

- Opening and closing items: The basic interaction techniques can be used to open and close items. Alternatively, a one-stroke gesture from an item to the tag above it can open it (Figure 4a) and another gesture from the item's top-right corner to its bottom-left corner can close it (Figure 4b).
- Views: The NFC-pinboard supports three views on its items: In the overview, all items are closed. In the standard view, items are either open or closed. The exposé presents all open items next to each other, similar to the homonymous Mac OS feature. Users can select any item to bring it to the foreground in the standard view. Users can switch between the views with options from the phone menu or the toolbar or by performing offset-gestures from one side of the NFCdisplay to the other.

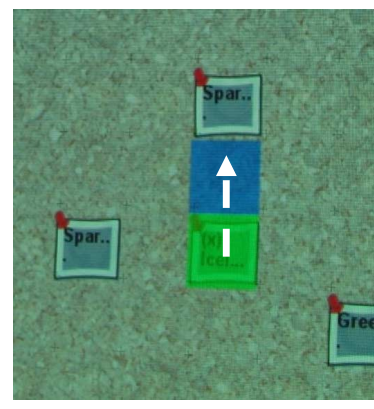

a)

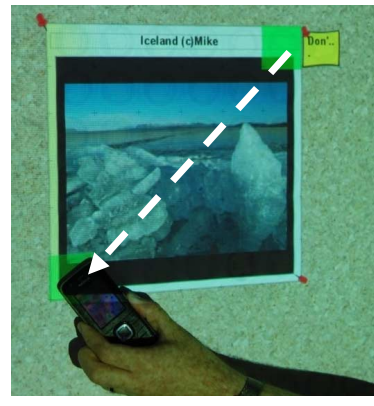

b)
Figure 4. Opening (a) and closing (b) items with gestures

- Tooltip: In order to get more information about closed items, users can apply a basic interaction technique to them (Figure 5a), similar to Hovering [5].

- Downloading items: Users can download items to their mobile devices with a basic interaction technique or a one-stroke gesture from the item to the tag beneath it (Figure 5b).

- Uploading items: Users can also create messages or pictures on their mobile devices and upload them to the NFC-pinboard by touching it. To upload an item as a closed icon, the target tag can be selected with a basic interaction technique. To upload an open item, users can define the target area with bounding-box-select.

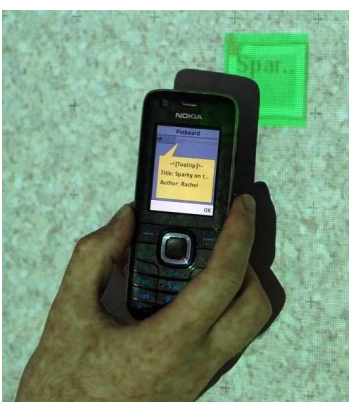

a)

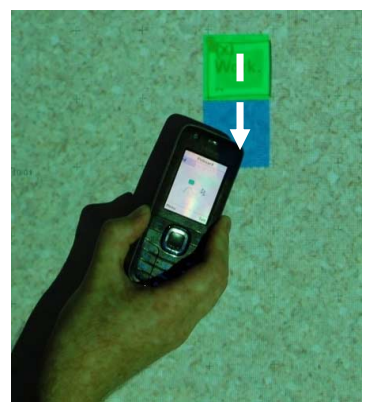

b)
Figure 5. Showing details about an item with the tooltip feature (a) and downloading an item (b) with a gesture

\section{Application-independent Interactions}

Apart from application-specific features, the prototype also supports interactions that are independent from the NFCpinboard and can also be used by other applications. 
- Selection of single and multiple items: The basic interaction techniques can be used to (de)select single and multiple items directly. For the indirect selection of items, the prototype supports polygon-select and bounding-box-select (Figure 6).
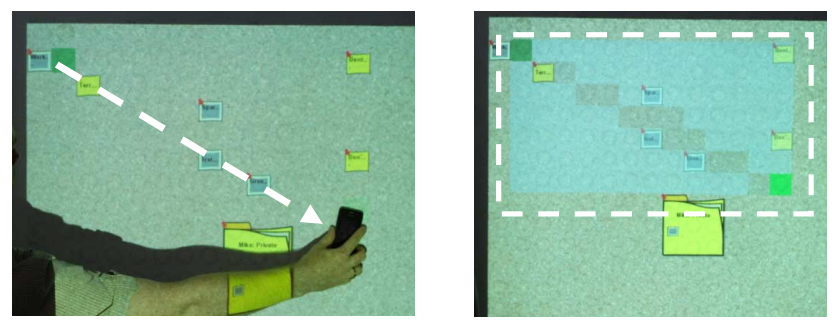

Figure 6. Selecting multiple items with bounding-box-select

- Context Menu: Each item has a context menu that can be opened with a basic interaction technique or a onestroke gesture from the item to the next tag on its right (Figure 7a). The menu comprises options to delete an item, to download and to move it (Figure 7b).

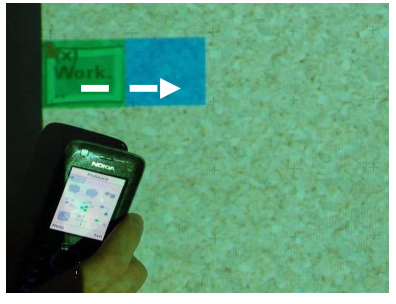

a)

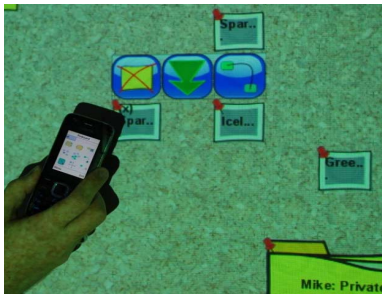

b)
Figure 7. Using a one-stroke gesture (a) to open the context menu of an item (b).

- Drag\&drop: Users can move items around the display with touch\&drop or an option from the context menu. Open items can also be picked up at their upper-left corner using touch-select (Figure 8).
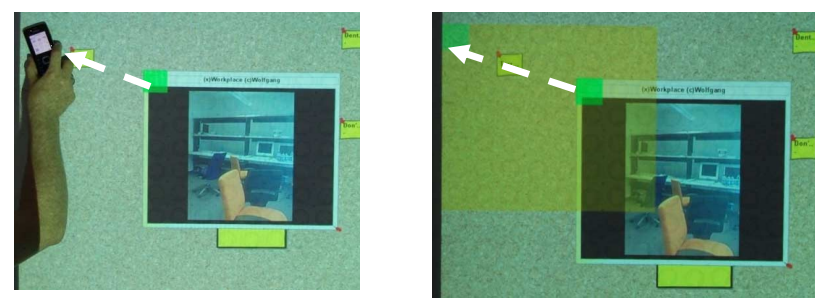

Figure 8. Moving an item by applying touch-select to its upper-left corner

\section{Technical Setup and Implementation}

The NFC-pinboard implements the conceptual hardwaresetup of dynamic NFC-displays (see Figure 1). The physical UI of the prototype is a poster with a grid of 20 by 15 adjacent NFC-tags, based on the Philips MiFare Ultralight standard, providing an interactive area of $90 \mathrm{~cm}$ by $67.5 \mathrm{~cm}$. The server application for the NFC-pinboard was implemented with Java SE and runs on a laptop. An LCD projector is used to project the application UI of the NFC-pinboard onto the physical UI.
The prototype is completed by a mobile Java ME application that runs on NFC-enabled mobile devices - in this case the Nokia 6212 mobile phone. Like other NFCenabled mobile phones that are currently available, it only supports discrete interactions with multiple tags. Therefore, the prototype does not support continuous interactions between tags of the grid but only discrete interactions with single tags. Gestures or shape-based input like polygonselect or the offset-shape-gestures are carried out as a series of discrete single-tag interactions (see discussion above). Apart from that, the phone serves as a smart pointing device to manipulate the projected application UI, to read the tags on the physical UI, to communicate with the application server via Bluetooth and to provide visual and vibration feedback during the interaction.

\section{USER STUDY AND EVALUATION}

The NFC-pinboard and its implementation of the interaction techniques allowed us to investigate their mapping to application features and to evaluate their applicability, usability and acceptance among users.

\section{Experimental Design and Tasks}

The study was conducted with 11 subjects (10 male, 1 female) with an average age of 26.6 years (from 23 to 33) that were recruited from our lab and a university. All subjects had a background in computer science and thus rated their general technical experience and their experience with mobile device with high averages of 6.3 and 5.1 on a Likert-scale from 1 ("inexperienced") to 7 ("experienced").

During the study, each subject had to carry out 8 tasks with the features of the NFC-pinboard. The tasks were designed to evaluate the applicability of different interaction techniques for specific application features. Therefore, each subject had to carry out each task with several different techniques and gestures. All subjects carried out the tasks in the same order because some of them built upon each other. The different interaction techniques and gestures that were tested during a task were used in random order.

- Tooltip: The subjects had to use touch-select, doubletouch, click-select, touch\&hold and options from the toolbar and the phone menu to get details about items (see Figure 5a).

- Opening and closing items: The subjects had to open and close items with touch-select, double-touch, clickselect, touch\&hold and gestures (see Figure 4).

- Switching views: The subjects had to switch between the views of the NFC-pinboard using options from the toolbar and the phone menu and performing offset gestures from one side of the NFC-display to the other.

- Uploading an item: The subjects had to upload an item from their mobile devices to the NFC-display using touch-select, click-select, touch\&hold, doubletouch and bounding-box-select. 
- Context menu: The subjects had to open the context menu of an item with touch-select, double-touch, clickselect, touch\&hold and a one-stroke gesture (see Figure 7a) and then select the delete-option with touch-select.

- Downloading an item: The subjects had to download an item to their mobile phones using touch-select, double-touch, click-select, touch\&hold, options from the toolbar and the phone menu and a one-stroke gesture (see Figure 5b).

- Drag\&drop: The subjects had to move items with the option from their context menus, touch\&drop based on touch-select and touch\&hold as well as touch-select with the dedicated upper-left corner of open items.

- Selection of multiple items: The subjects had to select multiple items directly and indirectly. The direct selection could be activated with an option from the toolbar or the phone menu and could be performed by selecting items with touch-select or touch\&hold. The indirect selection was done by drawing a bounding-box around items using touch-select or touch\&hold.

During the study, we collected qualitative and quantitative data to evaluate the different interaction techniques, their usability, their mapping to application features and the preferences of the subjects. The subjects were recorded on video for a post-hoc analysis of errors and attention shifts from mobile devices to the NFC-display or vice versa.

\section{Results}

\section{User Preferences}

After each task, the subjects had to fill out a questionnaire to assess which interaction technique or gesture they liked the best and the least for the tested feature of the NFCpinboard. They also had to rate different properties of the gestures on a Likert-scale from 1 ("fully disagree") to 7 ("totally agree").

- Tooltip: Most subjects (7) preferred touch-select for this feature and regarded it as fast, simple and intuitive. Two subjects preferred the option from the toolbar and one subject voted for click-select and touch\&hold each. The least preferred interaction techniques were clickselect (4), the phone menu (3) and double-touch (3) because of their added complexity which did not fit the simplicity of the feature. Double-touch caused problems because of the delay between its two touches.

- Opening and closing items: For this feature, five subjects preferred touch-select because of its speed and simplicity. Two subjects preferred the one-stroke gestures and three subjects favored click-select because they felt more in control with it and appreciated the explicit triggering of actions. On the other hand, three subjects disliked click-select because of the added attention shift. Interestingly, two subjects rejected touch-select as too simple for this feature. Three subjects each did not like touch\&hold and the gestures and thought that they were tedious and intricate. Consequently, the subjects rated the gestures as very easy to learn $($ mean $=6.5$; standard deviation $=0.5)$ and easy to use $(\mathrm{m}=5.9 ; \mathrm{sd}=0.7)$, but neither as very fast $(\mathrm{m}=4.2 ; \mathrm{sd}=0.8)$ or effective $(\mathrm{m}=4.5 ; \mathrm{sd}=1.8)$.

- Switching views: Eight subjects liked the toolbar and its options to switch between the views and regarded it as fast and easy to use. Three subjects preferred the phone to switch the views because it gave them a better overview of the NFC-display. Three subjects disliked the same technique because of the added attention shifts. Six subjects disliked the gestures from one side of the display to the other, which were difficult to carry out. Nevertheless, the gestures received good ratings for ease of learning $(\mathrm{m}=5.9 ; \mathrm{sd}=1.2)$ and ease of use $(\mathrm{m}=5.0 ; \mathrm{sd}=1.4)$, but not for speed $(\mathrm{m}=3.7 ; \mathrm{sd}=$ $1.0)$ and effectiveness $(\mathrm{m}=3.9 ; \mathrm{sd}=1.7)$.

- Uploading items: For this feature, most subjects preferred touch-select (5) or bounding-box-select (4). The former was liked for its speed, simplicity and intuitiveness, the latter for the possibility to define the size of an open item. Click-select (7) and double-touch (3) were the least liked interaction techniques because of their added complexity compared to touch-select, respectively the additional touch-input (double-touch) and attention shifts (click-select).

- Context menu: For this feature, five subjects preferred the one-stroke gesture because it was easy to distinguish from other techniques. It was also very easy to learn $(\mathrm{m}=6.6 ; \mathrm{sd}=0.5)$ and to use $(\mathrm{m}=6.4 ; \mathrm{sd}=$ $0.8)$, as well as fast $(\mathrm{m}=5.8 ; \mathrm{sd}=1.0)$ and effective $(\mathrm{m}$ $=6.0 ; \mathrm{sd}=0.9)$. Three subjects liked touch\&hold which seemed familiar from interactions with other applications. The remaining three subjects voted for touch-select because of its simplicity. On the other hand, three subjects each regarded touch\&hold, double-touch and click-select as the least suitable technique for this feature. Compared to touch-select, touch\&hold was too slow and not accurate enough. Double-touch was rejected because of the delay between the single touch-selects. Click-select was seen as uncomfortable.

- Downloading items: For this feature, five subjects preferred the one-stroke gesture, which was regarded as intuitive, very easy to learn $(\mathrm{m}=6.5 ; \mathrm{sd}=0.5)$ and use $(\mathrm{m}=6.3 ; \mathrm{sd}=0.8)$, fast $(\mathrm{m}=5.9 ; \mathrm{sd}=0.7)$ and effective $(\mathrm{m}=6.1 ; \mathrm{sd}=0.8)$. Three subjects liked the option from the context menu, which was familiar from PCs and not easy to be confused with other techniques. The context menu was disliked by three subjects because it was too slow. Touch\&hold and touch-select were rejected by two subjects each. Touch\&hold was regarded as too imprecise and touch-select could be 
performed too easily and cause accidental and unwanted downloads.

- Drag\&drop: Five subjects preferred touch\&drop with touch-select to move items around the display. Four subjects preferred the interaction with the upper-left corner of items. Both techniques were regarded as intuitive, fast and easy to use. Two subjects preferred touch\&drop with touch\&hold as it gave them a better feeling of picking items up. The least preferred technique for drag\&drop was the option from the context menu (6), which was too complicated, not intuitive and required too many steps. Three subjects disliked touch-select with the corner of the item because it could only be performed with open items. Two subjects disliked touch\&drop with touch\&hold because of its delay.

- $\quad$ Selection of multiple items: Seven subjects preferred the indirect selection of items with bounding-boxselect using touch-select because it was simple, fast and familiar from similar desktop interactions. Two subjects liked the activation of multi-select from the toolbar because it allowed them to select single items with touch-select. Most subjects disliked the combination of touch\&hold with the phone menu (6) or the toolbar (4). Switching to multi-select via the phone menu was regarded as very uncomfortable.

\section{Errors and Attention Shifts}

We complemented the qualitative evaluation of interaction techniques and gestures with quantitative data about errors and attention shifts for the most common interaction techniques. Touch\&drop and the selection of multiple items were not included in this evaluation because they employ more specialized interaction techniques. A caveat for the interpretation of this data is the learning effects that result from the fixed order of the tasks. They were not randomized or counter-balanced because they partly built upon each other. The interaction techniques and gestures that were tested within a task were randomized.

Figure 9 shows the mean numbers of errors for the most common interaction techniques that were tested for the different application features. It shows the learning curve as the number of errors for most interaction techniques and the total number of errors for each feature decrease during the study. During the first task with the tooltip-feature, the subjects made quite a lot of errors with every interaction technique but quickly became better. Towards the end of the study, they had gotten used to most interaction techniques and made hardly any errors, which is shown by the results for the download-task. Figure 9 also indicates that apart from the combination of gestures and views, double-touch and click-select caused the most errors $(0.35$ and 0.24 on average), followed by touch\&hold $(0.20)$, touch-select $(0.18)$, the toolbar $(0.16)$ and the phone menu
(0.12). These average values are only indicative, as the techniques were not equally tested across all features.

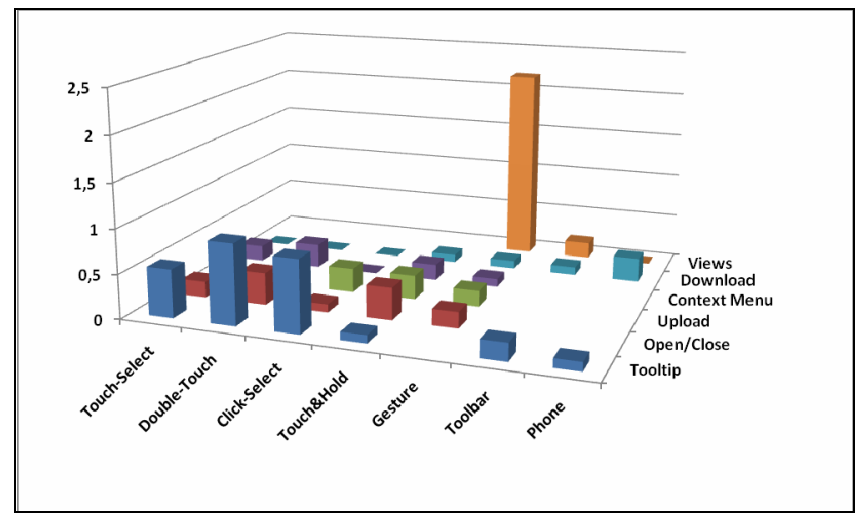

Figure 9. Overview of the mean numbers of errors for interaction techniques and application features

The mean numbers of attention shifts in Figure 10 do not show a consistent learning curve, because some features cause them almost automatically. For example, users often look from the NFC-display to their mobile devices when they perform a click-select, which often causes high numbers of attention shifts during different tasks. Together with the phone menu, click-select causes the highest total average of attention shifts (3.93 vs. 3.62). Touch-select, double-touch, touch\&hold and the toolbar cause about the same total average of attention shifts $(2.11,2.20,2.31$, $2.25)$, outplayed by gestures which cause only 1.35 attention shifts on average.

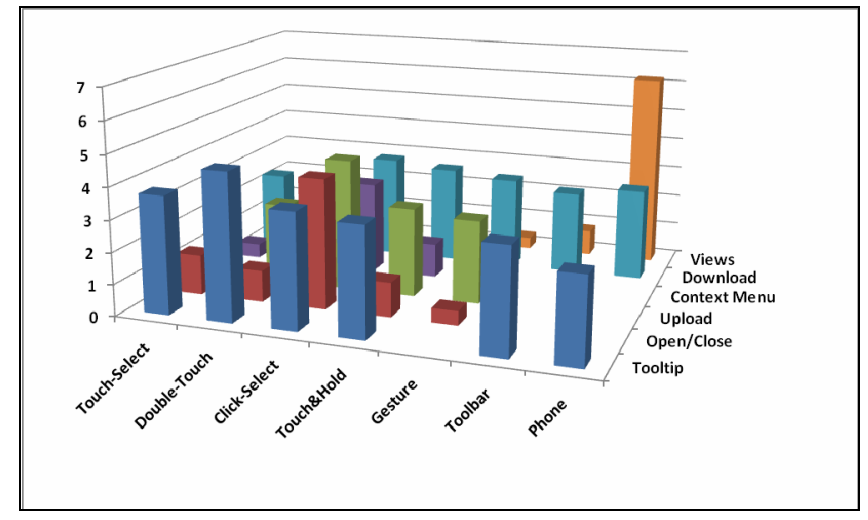

Figure 10. Overview of the mean numbers of attention shifts for interaction techniques and application features

\section{SUMMARY AND CONCLUSION}

In this paper, we have presented the design, application and evaluation of interaction techniques for dynamic NFCdisplays. Based on the capabilities of this technology, we have partially adopted interaction techniques from related work and have created new techniques like touch\&hold, double-touch or discrete gestures. We have implemented these techniques with an NFC-pinboard prototype in order to evaluate their mapping to specific application features in a comprehensive user study. Apart from their applicability 
for different application features, we have also investigated their usability, (dis)advantages and user acceptance.

Some results of the user study are not surprising: The evaluation of touch-select confirms the simplicity of touching a single tag as the greatest advantage of mobile interaction with NFC, making it the preferred technique for all kinds of interactions and application features. However, some results also indicate that touch-select might actually be too simple for interactions for which some users prefer a feeling of being in control, e.g. triggering or confirming important actions with the familiar press of a button or avoiding accidental actions.

Disregarding the general appreciation of touch-select, the more interesting results are provided by the evaluation of the other interaction techniques: Double-touch, touch\&hold and click-select did not perform too well. Many subjects disliked the additional time and effort that was required by these techniques to touch another tag, to press a key or to shift one's attention on top of touch-select. However, the results indicate that this effort can be accepted for interactions for which touch-select is too simple and lightweight. Although these interaction techniques are not as simple, intuitive or popular as touch-select, they can be used to diversify this atomic interaction technique for applications that require different techniques to implement their different features.

Furthermore, the comparison of menus showed that the subjects prefer the simple interaction with the toolbar and dislike the interaction with the uncomfortable phone menu. While the context-menu was seen as an opportunity to avoid interference with other interactions, it also caused additional effort. The gestures were well-received and seen as alternatives for other techniques as long as they were easy to perform. The subjects preferred short gestures that were applied to items and disliked gestures that required them to interact across the whole display.

With our work on interaction techniques for dynamic NFCdisplays, we also tried to push the limits of mobile interaction with NFC and what is possible with this technology. Current limitations and challenges for future work in this area include the interaction with smaller and with multiple tags, the continuous selection of tags to improve gestures and shape-based input or the combination of NFC and other technologies, e.g. sensor data.

Although dynamic NFC-displays are only a prototyping technology, they anticipate technologies for more direct and physical interactions between mobile devices and displays. Our work on interaction techniques for this technology can provide preliminary results to inform the design and development of future mobile interactions with NFC-based physical UIs. The results of our work show that dynamic NFC-displays have a potential for interaction techniques and even discrete gestures beyond touch-select.

\section{REFERENCES}

1. Ballagas, R., Rohs, M., and Sheridan, J. G. 2005. Sweep and point and shoot: phonecam-based interactions for large public displays. In: CHI '05 extended abstracts. 2005, ACM Press.

2. Broll, G., Rukzio, E., Paolucci, M., Wagner, M., Schmidt, A., and Hussmann, H. 2009. Perci: Pervasive Service Interaction with the Internet of Things. IEEE Internet Computing 13, 6 (Nov. 2009), 74-81.

3. Boring, S., Jurmu, M., and Butz, A. 2009. Scroll, tilt or move it: using mobile phones to continuously control pointers on large public displays. In Proc. of OZCHI '09, ACM, 161-168.

4. Ferscha, A. and Vogl, S. 2002. Pervasive Web Access via Public Communication Walls. In: Proc. of Pervasive 2002 F. Mattern and M. Naghshineh (Eds.). Lecture Notes In Computer Science. vol. 2414. Springer-Verlag, 84-97.

5. Hardy, R. and Rukzio, E. 2008. Touch \& interact: touch-based interaction of mobile phones with displays. In Proc. of MobileHCI '08. ACM, 245-254.

6. Li, Y., Hinckley, K., Guan, Z., and Landay, J. A. 2005. Experimental analysis of mode switching techniques in penbased user interfaces. In Proc. of CHI '05, ACM, 461-470.

7. NTT DOCOMO i-mode Felica, www.nttdocomo.co.jp/ english/service/imode/make/content/felica/index.html

8. Oyster Card website: https://oyster.tfl.gov.uk/oyster/entry.do

9. Ramírez-González, G., Muñoz-Organero, M., Kloos, C. D., and Astaiza, Á. C. 2008. Exploring NFC interactive panel. In Proc. of Mobiquitous 2008. July 21 - 25, 2008.

10. Reilly, D., Welsman-Dinelle, M., Bate, C., and Inkpen, K. 2005. Just point and click?: using handhelds to interact with paper maps. In Proc. of MobileHCI '05, ACM, 239-242.

11. Rekimoto, J. 1997. Pick-and-drop: a direct manipulation technique for multiple computer environments. In Proc. of UIST '97. ACM, 31-39.

12. Riekki, J., Salminen, T., and Alakärppä, I. 2006. Requesting Pervasive Services by Touching RFID Tags. IEEE Pervasive Computing 5(1): 40-46.

13. Sánchez, I., Riekki, J., and Pyykknen, M. 2008. Touch \& control: Interacting with services by touching RFID tags. In Proc. of IWRT 08, June 12-13 2008.

14. Schwieren, J. and Vossen, G. 2007. Implementing Physical Hyperlinks for Mobile Applications Using RFID Tags. In Proc. of IDEAS`07. IEEE Computer Society, 154-162.

15. Seewoonauth, K., Rukzio, E., Hardy, R., and Holleis, P. 2009. Touch \& connect and touch \& select: interacting with a computer by touching it with a mobile phone. In Proc. of MobileHCI '09. ACM, 1-9.

16. SmartTouch website. www.smarttouch.org

17. Vetter, J., Hamard, J., Paolucci, M., Rukzio, E., and Schmidt, A. 2007. Physical Mobile Interaction with Dynamic Physical Objects. Demo at MobileHCI'07, September 9, 2007.

18. Want, R., Fishkin, K. P., Gujar, A., and Harrison, B. L. 1999. Bridging physical and virtual worlds with electronic tags. In Proc. of CHI '99. ACM, 370-377.

19. Want, R. 2006. An Introduction to RFID Technology. IEEE Pervasive Computing 5, 1 (Jan. 2006), 25. 